\title{
Investigating Unusual Homonuclear Intermolecular "Through-Space" J Couplings in Organochalcogen Systems
}

\author{
Paula Sanz Camacho, ${ }^{\dagger}$ David McKay, ${ }^{\dagger}$ Daniel M. Dawson, ${ }^{\dagger}$ Christin Kirst, ${ }^{\dagger}$ Jonathan R. Yates, ${ }^{\ddagger}$ \\ Timothy F. G. Green, ${ }^{\ddagger}$ David B. Cordes, ${ }^{\dagger}$ Alexandra M. Z. Slawin, ${ }^{\dagger}$ J. Derek Woollins, ${ }^{*}{ }^{\dagger}$ \\ and Sharon E. Ashbrook ${ }^{*} \dagger$ \\ ${ }^{\dagger}$ School of Chemistry, EaStCHEM and Centre of Magnetic Resonance, University of St Andrews, St Andrews, Fife KY16 9ST, U.K. \\ ${ }^{\ddagger}$ Department of Materials, University of Oxford, Oxford OX1 3PH, U.K.
}

\section{Supporting Information}

\begin{abstract}
Although the electron-mediated spin-spin or J coupling is conventionally viewed as transmitted via covalent bonds, examples of $\mathrm{J}$ couplings between atoms that are not formally bonded but are in close proximity (termed "throughspace" J couplings) have been reported. In this work, we investigate the observation of homonuclear ${ }^{31} \mathrm{P} \mathrm{J}$ couplings in organochalcogen heterocycles, which occur between ${ }^{31} \mathrm{P}$ in two separate molecules, confirming without doubt their throughspace nature. The presence of this interaction is even more surprising for one compound, where it occurs between crystallographically equivalent species. Although crystallographically equivalent species need not be magnetically

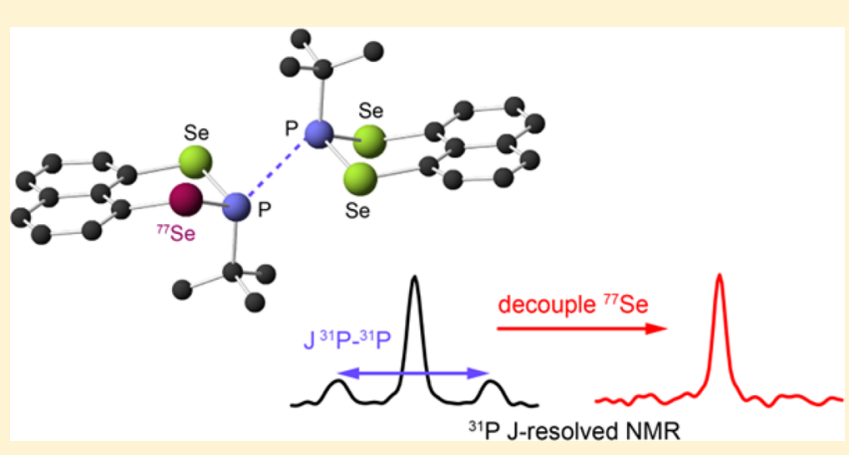
equivalent in the solid state, owing to the presence of anisotropic interactions, we demonstrate that it is not the shielding anisotropy that lifts magnetic equivalence, in this case, but the presence of heteronuclear couplings to ${ }^{77} \mathrm{Se}$. We support our experimental observations with periodic scalar-relativistic density functional theory calculations and coupling density deformation plots to visualize the mechanism of these interesting interactions.
\end{abstract}

\section{INTRODUCTION}

Indirect spin-spin (J) couplings are invaluable for the determination of chemical structure in solution-state NMR spectroscopy. ${ }^{1}$ This electron-mediated coupling is classically considered to be transmitted via a network of covalent bonds, generally decreasing in magnitude as the number of bonds increases. However, in some cases, J couplings are observed between atoms that are not formally bonded but are in close proximity (generally termed "through-space" J couplings in the literature), suggesting that a more sophisticated description of the interaction is required. ${ }^{2-5}$ The vast majority of these observations, summarized recently in ref 2 , involve atoms that are held close in space by rigid molecular architectures, such as $\mathrm{H}, \mathrm{C}$, and F in cyclic and organic molecules, and F, P, Se, and $\mathrm{Te}$ in peri-substituted naphthalene, polyaromatic, or metallocene-based structural motifs. ${ }^{2-7}$ Such couplings have been used in structure solution, that is, to determine molecular conformations in solution or to measure internuclear distances, but have also been studied with a view to gaining a more fundamental understanding of their exact nature and mechanism.

The rapid tumbling motion and low concentration of molecules present in a solution typically restricts the observation of through-space couplings to those that exist within the same molecule. In many cases, some uncertainty then remains over the relative magnitude of any through-bond contribution to the interaction, unless a very large number of bonds separate the species. In the solid state, however, it should, in principle, be possible to see intermolecular throughspace J couplings, that is, between different molecules, if they are held in close proximity by the crystal packing. The existence of these interactions, and their relative magnitude in simple systems, has been studied computationally. ${ }^{5,-10}$ However, there remain relatively few experimental reports of any throughspace $\mathrm{J}$ couplings in solids, either intermolecular or intramolecular, in the literature, ${ }^{11-16}$ although some J couplings through intermolecular hydrogen bonding interactions have been observed. ${ }^{17}$ Examples of intramolecular through-space couplings observed in the solid state have included the observation of ${ }^{77} \mathrm{Se}-{ }^{125} \mathrm{Te}$ and ${ }^{31} \mathrm{P}-{ }^{125} \mathrm{Te}$ couplings in perisubstituted naphthalenes, ${ }^{14,15}{ }^{11} \mathrm{~B}-{ }^{31} \mathrm{P}$ interactions in frustrated Lewis pairs in alkenes, ${ }^{12}$ and ${ }^{31} \mathrm{P}-{ }^{31} \mathrm{P}$ interactions in ferrocenes. ${ }^{11}$ In general, J couplings can be more difficult to resolve directly in the solid state, owing to the larger inherent spectral line widths that are typically present and, while they are still used for magnetization transfer in multidimensional correlation experiments, it can then be difficult to distinguish

Received: May 7, 2016

Published: July 17, 2016 
between cross peaks arising from $\mathrm{J}$ couplings and those from residual dipolar (i.e., true through-space) interactions (which have no isotropic component). In 2015, Sanz Camacho et al. ${ }^{18}$ observed an intermolecular $\mathrm{J}$ coupling in a $\mathrm{P}-\mathrm{Se}$ heterocyclic molecule. The multiplet structure in the ${ }^{77} \mathrm{Se}$ spectrum suggested the presence of two distinct couplings to ${ }^{31} \mathrm{P}$, a result confirmed by ${ }^{31} \mathrm{P}$ decoupling. As there is only one $\mathrm{P}$ per molecule, the second coupling has, by definition, to be to ${ }^{31} \mathrm{P}$ in an adjacent molecule, confirming the lack of any conventional bonding pathway between the spins. This observation was supported by periodic DFT calculations, which predicted two ${ }^{31} \mathrm{P}-{ }^{77} \mathrm{Se} \mathrm{J}$ couplings, one through space and one through bond, with very similar magnitudes. A similar heteronuclear $\left({ }^{119} \mathrm{Sn}-{ }^{31} \mathrm{P}\right)$ intermolecular coupling has subsequently been observed by Arras et al. ${ }^{19}$

In this work, we demonstrate the observation (in organochalogen heterocycles) of some of the first, if not the first, intermolecular homonuclear through-space $\mathrm{J}$ couplings in solids. In one compound the coupling can be observed directly in the ${ }^{31} \mathrm{P}$ spectrum, while in a second it is not directly resolved, but can be measured using J-resolved spectroscopy. This latter interaction is of particular note as it occurs between two crystallographically equivalent species, and we show that magnetic equivalence is lifted only by the presence of heteronuclear $\mathrm{J}$ couplings to ${ }^{77} \mathrm{Se}$, allowing experimental measurement. Unlike the previously observed heteronuclear interactions, it is not possible to easily remove homonuclear $\mathrm{J}$ couplings using decoupling, and so we support our conclusions using periodic relativistic density functional theory (DFT) calculations and exploit coupling density deformation (CDD) ${ }^{20}$ plots to visualize the mechanism by which these interesting interactions take place. These observations add to the rich diversity of weak interactions that contribute to crystal packing and are of particular importance for chalcogen-rich molecular materials, which contain large and polarizable atoms. It is hoped that the additional insight gained from this work can offer further advances in the future design of solid-state structures.

\section{EXPERIMENTAL AND COMPUTATIONAL METHODS}

Nuclear Magnetic Resonance Spectroscopy. Solid-state ${ }^{31} \mathrm{P}$ NMR measurements were performed using Bruker Avance III spectrometers equipped with either 9.4, 14.1, or $20.0 \mathrm{~T}$ wide-bore superconducting magnets. Experiments were performed using conventional $4 \mathrm{~mm}$ magic-angle spinning (MAS) probes, with MAS rates between 5 and $12.5 \mathrm{kHz} .{ }^{31} \mathrm{P}$ chemical shifts are quoted relative to $85 \%$ $\mathrm{H}_{3} \mathrm{PO}_{4}$ using $\mathrm{BPO}_{4}$ at $-29.6 \mathrm{ppm}$ as a secondary reference. For $\mathbf{1}$, spectra were acquired using cross-polarization (CP) from ${ }^{1} \mathrm{H}$ with a contact pulse (ramped for ${ }^{1} \mathrm{H}$ ) of $1 \mathrm{~ms}$ and are the result of averaging 256 transients, with a typical recycle interval of $10 \mathrm{~s}$. For 2 and 3, spectra were acquired directly, averaging between 32 and 64 transients, respectively, with a typical recycle interval of $30 \mathrm{~s}$. Continuous wave (cw) ${ }^{1} \mathrm{H}$ decoupling $\left(\gamma \mathrm{B}_{1} / 2 \pi \approx 100 \mathrm{kHz}\right)$ was applied during acquisition for 1-3 but not for the variable-temperature experiments, as no significant changes in resolution were observed. The positions of the isotropic resonances within the spinning sideband manifold were unambiguously determined by recording a second spectrum at a different MAS rate. Chemical shift anisotropy (CSA) parameters were determined by line shape analysis using the Bruker Topspin software. Two-dimensional ${ }^{31} \mathrm{P}$ homonuclear J-resolved spectra were acquired at 9.4, 14.1, and $20.0 \mathrm{~T}$ with a MAS rate of $5 \mathrm{kHz}$, using the pulse sequence given in ref 21 . Owing to the long ${ }^{31} \mathrm{P}$ longitudinal relaxation time for $1, \mathrm{CP}$ from ${ }^{1} \mathrm{H}$ was used with a $1 \mathrm{~ms}$ contact pulse (ramped for ${ }^{1} \mathrm{H}$ ). For $\mathbf{2}$ and $\mathbf{3}$, transverse magnetization was created directly. In each case, between 32 and 64 transients were averaged for each of
256-320 $t_{1}$ increments of $800 \mu \mathrm{s}$. ${ }^{1} \mathrm{H}$ decoupling (cw, $\gamma \mathrm{B} 1 / 2 \pi$ of $\sim 100$ $\mathrm{kHz}$ ) was applied in both $t_{1}$ and $t_{2}$. For 2 , a spectrum was also acquired at $14.1 \mathrm{~T}$ using rotor-synchronized Hahn-echo pulse train ${ }^{22}$ decoupling of ${ }^{77} \mathrm{Se}\left(\gamma \mathrm{B}_{1} / 2 \pi\right.$ of $\left.\sim 62.5 \mathrm{kHz}\right)$ during $t_{1}$, and ${ }^{1} \mathrm{H}$ decoupling ( $\mathrm{cw}, \gamma \mathrm{B}_{1} / 2 \pi$ of $\sim 100 \mathrm{kHz}$ ) was applied in both $t_{1}$ and $t_{2} .64$ transients were averaged for each of $256 t_{1}$ increments of $800 \mu \mathrm{s}$. For controlled-temperature experiments recorded at 9.4 and $14.1 \mathrm{~T}$, the sample temperature was maintained at 273,298 , or $323 \mathrm{~K}$ using a Bruker BCU-II chiller and Bruker BVT/BVTB-3000 temperature controller and heater booster. The sample temperature (including frictional heating effects arising from sample spinning) was calibrated using the isotropic ${ }^{87} \mathrm{Rb}$ shift of solid $\mathrm{RbCl} .{ }^{23}$

$\mathrm{A}^{13} \mathrm{C}(9.4 \mathrm{~T}, 12.5 \mathrm{kHz}) \mathrm{CP}$ MAS NMR spectrum of 1 was acquired with a contact pulse (ramped for ${ }^{1} \mathrm{H}$ ) duration of $8 \mathrm{~ms}$. Signal averaging was performed for 1072 transients, with a recycle interval of $10 \mathrm{~s} .{ }^{13} \mathrm{C}$ chemical shifts are quoted in parts per million relative to $\left(\mathrm{CH}_{3}\right)_{4} \mathrm{Si}$, using the $\mathrm{CH}_{3}$ resonance of L-alanine $(\delta=20.5 \mathrm{ppm})$ as a secondary reference.

Calculations. Calculations were performed using the CASTEP ${ }^{24,25}$ DFT code (version 8.0), using the $\mathrm{PBE}^{26}$ exchange-correlation functional, with dispersion effects accounted for through the DFTD2 scheme of Grimme. ${ }^{27,28}$ Atomic positions and unit cell parameters were optimized from crystallographically determined data. CASTEP 8.0 default on-the-fly ultrasoft pseudopotentials were used, with the inclusion of scalar-relativistic effects through ZORA. ${ }^{29}$ A planewave basis set with $E_{\text {cut }}$ of 50 Ry was used, and a Monkhurst-Pack grid with spacing of $0.042 \pi \AA^{-1}$ was used to sample the Brillouin zone. $E_{\text {cut }}$ and k-point spacing were converged independently against total energy and $\sigma_{\text {iso }}$ for nuclei of interest, as described in the Supporting Information. NMR calculations were performed using GIPAW after geometry optimization (see the Supporting Information). PAW J-coupling calculations are based upon $2 \times 1 \times 1$ supercell geometries. Calculations were performed on a local cluster, with the exception of the supercell J-coupling calculations for $\mathbf{1}$, which were performed on the ARCHER U.K. National Supercomputing Service. Calculations generate the absolute shielding tensor $\sigma$ in the crystal frame. Diagonalization of the symmetric part of $\sigma$ yields three orthogonal principal components, namely, $\sigma_{11}, \sigma_{22}$, and $\sigma_{33}$, with $\sigma_{33} \geq \sigma_{22} \geq \sigma_{11}$. The isotropic shielding value $\sigma_{\text {iso }}$ is given by $\left(\sigma_{11}+\sigma_{22}+\sigma_{33}\right) / 3$. The anisotropy is defined by the span, $\Omega=\sigma_{33}-\sigma_{11}$, and the skew $\kappa=$ $3\left(\sigma_{\text {iso }}-\sigma_{22}\right) / \Omega$ is a measure of the asymmetry of the tensor in the Herzfeld-Berger convention. For further information see the Supporting Information.

\section{RESULTS AND DISCUSSION}

Scheme 1 shows the synthetic route for the preparation of the organochalcogen heterocycles. Naphtho[1,8-cd]-1,2-dithiole

Scheme 1. General Synthesis Scheme for Compounds 1-3

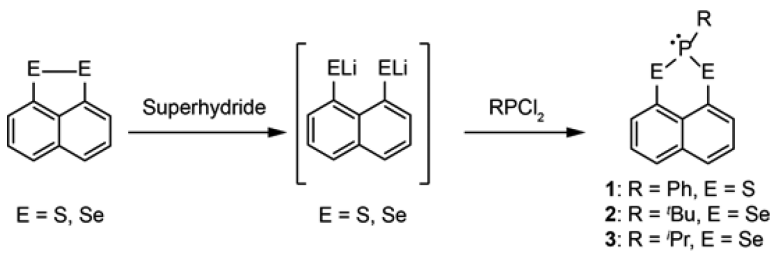

phenylphosphine (1) was prepared according to ref 30 and characterized using IR spectroscopy, solution-state NMR spectroscopy, mass spectrometry, and single-crystal X-ray diffraction, as described in the Supporting Information. Naphtho[1,8-cd]-1,2-diselenole tert-butylphosphine (2) and naphtho[1,8-cd]-1,2-diselenole isopropylphosphine (3) were prepared and characterized as described in recent work. ${ }^{18}$

Naphtho[1,8-cd]-1,2-dithiole phenylphosphine (1). The ${ }^{31} \mathrm{P}$ MAS NMR spectra of $\mathbf{1}$, acquired at two different fields using $\mathrm{CP}$ from ${ }^{1} \mathrm{H}$ (owing to the long ${ }^{31} \mathrm{P} \mathrm{T}_{1}$ relaxation 
time), are shown in Figure 1a. A significant sideband manifold is observed as a result of the CSA, with the position of the
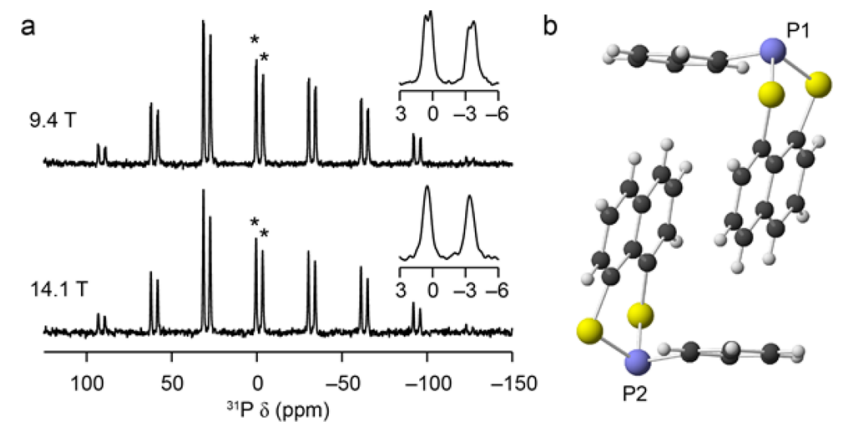

Figure 1. (a) ${ }^{31} \mathrm{P}$ NMR spectra of $\mathbf{1}$ recorded at $9.4 \mathrm{~T}, 5 \mathrm{kHz}$ CP MAS (top), and 14.1 T, 7.5 kHz CP MAS (bottom). In both spectra, the isotropic center bands are marked with * and expanded in the inset. (b) The asymmetric unit of $\mathbf{1}$.

isotropic center bands (determined by acquiring a second spectrum at a faster MAS rate) shown by *. Isotropic and anisotropic shielding parameters are given in Table 1 . Note that

Table 1. Experimental ${ }^{31} P$ Solid-State NMR Parameters ${ }^{a}$ for 1-3

$\begin{array}{lrrrr}\text { compound } & \delta_{\text {iso }}(\mathrm{ppm}) & \Omega(\mathrm{ppm}) & \kappa & J_{\mathrm{PP}}, \mathrm{Hz} \\ \mathbf{1}(\mathrm{P} 1) & 0.6(1) & 197(2) & 0.25(5) & 86(2) \\ \mathbf{1}(\mathrm{P} 2) & -3.3(1) & 193(2) & 0.23(5) & 86(2) \\ \mathbf{2} & 6.1(1) & 231(3) & 0.50(5) & 88(2) \\ \mathbf{3} & -2.1(2) & 197(3) & 0.40(5) & \end{array}$

${ }^{a}$ Isotropic chemical shift, $\delta_{\text {iso }}$ span, $\Omega$, skew, $\kappa$, and intermolecular J coupling, $J_{\mathrm{Pp}}$. Measured at $B_{0}=14.1 \mathrm{~T}, \mathrm{MAS}$ rate $=7.5 \mathrm{kHz}, T=298$ $\mathrm{K}$.

the NMR parameters show a small temperature dependence (i.e., a change of $0.7 \mathrm{ppm}$ in the isotropic shift per $25 \mathrm{~K}$ ), as shown in the Supporting Information, probably resulting from motion of the phenyl group.

Two ${ }^{31} \mathrm{P}$ signals are observed for $\mathbf{1}$, in agreement with the single-crystal diffraction structure, which shows two crystallographically distinct molecules in the asymmetric unit, as shown in Figure 1b. A low-level (unknown) impurity phase is also present. Shielding parameters calculated using a periodic planewave DFT approach ${ }^{24,25}$ are given in Table 2. The calculations enable assignment of the two ${ }^{31} \mathrm{P}$ resonances, with that at higher shift $(0.6 \mathrm{ppm})$ attributed to $\mathrm{P} 1$ and that at lower shift $(-3.3 \mathrm{ppm})$ to P2. The shift difference between the two is overestimated by the calculations, as are the values of $\Omega$, although the two are predicted to be extremely similar, as is also

Table 2. Calculated ${ }^{31} \mathrm{P}$ Solid-State NMR Parameters ${ }^{a}$ for $1-$ 3

$\begin{array}{lcccc} & \sigma_{\text {iso }}^{\text {calc }}(\mathrm{ppm}) & \Omega^{\text {calc }}(\mathrm{ppm}) & \kappa^{\text {calc }} & J_{\mathrm{PP}}^{\text {calc }}, \mathrm{Hz} \\ \mathbf{1}(\mathrm{P} 1) & 281(281) & 259(259) & 0.26(0.27) & 151(144) \\ \mathbf{1}(\mathrm{P} 2) & 290(289) & 259(259) & 0.26(0.26) & 151(144) \\ \mathbf{2} & 244(254) & 311(317) & 0.39(0.40) & 159(155) \\ \mathbf{3} & 251(260) & 267(275) & 0.17(0.21) & 11(12)\end{array}$

${ }^{a}$ Isotropic chemical shielding, $\sigma_{\text {iso }}^{\text {calc }}$, span, $\Omega^{\text {calc }}$, skew, $\kappa^{\text {calc }}$, and intermolecular $\mathrm{J}$ coupling, $J_{\mathrm{PP}}^{\text {calc. }}$. Scalar relativistic and non-relativistic values are given, with the latter in parentheses. seen experimentally. Little difference is observed upon the inclusion of scalar relativity (using the ZORA approach). The difference between calculation and experiment probably results from the neglect of motion in the DFT calculations, which are performed on static structures at $0 \mathrm{~K}$. The spectrum acquired at 9.4 $\mathrm{T}$ exhibits small splittings in each of the resonances, possibly indicative of $\mathrm{J}$ couplings, although the presence of an additional impurity phase (or polymorph), commonly observed in these types of systems, cannot be ruled out at this stage. Owing to the large inherent line widths in solid-state NMR spectra, J couplings are often not observed directly and are more typically measured using the modulation of the signal intensity in a spin-echo experiment. Fourier transformation as a function of the echo evolution period gives a so-called "Jresolved" spectrum, where inhomogeneities are refocused. A detailed theoretical study by Duma et al. ${ }^{31}$ investigated how the homonuclear echo modulation was affected by experimental conditions, including the magnetic field strength and MAS rate, and by the magnitude and orientation of the spin interaction tensors. Although this was shown to be a complex problem, for most practical conditions, a simple modulation was predicted, and the J coupling could easily be extracted from the spectral splitting.

Figure 2 shows a two-dimensional ${ }^{31} \mathrm{P}$ homonuclear $\mathrm{J}$ resolved spectrum (isotropic center bands only) of $\mathbf{1}$, along

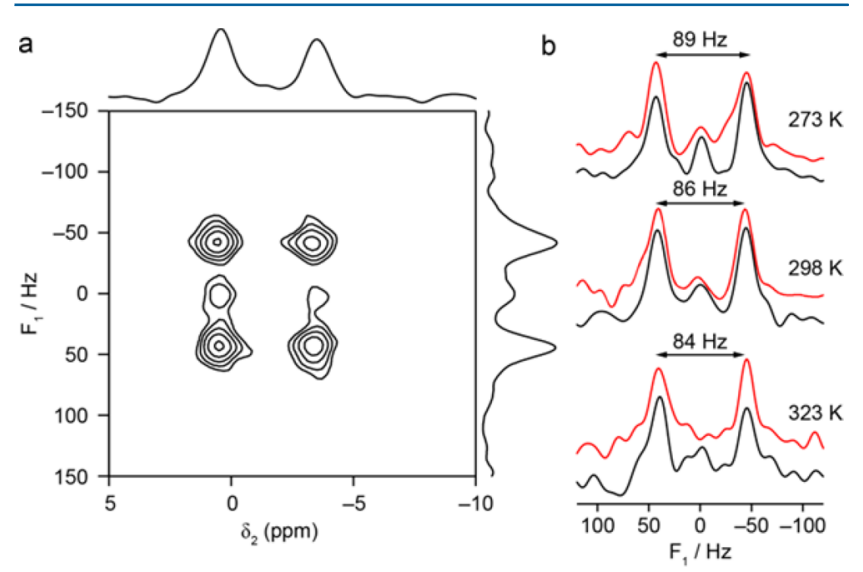

Figure 2. (a) Expansion (showing the isotropic center bands) from the two-dimensional homonuclear ${ }^{31} \mathrm{P}$ J-resolved spectrum of 1 (14.1 T, 5 $\mathrm{kHz}$ CP MAS). ${ }^{1} \mathrm{H}$ decoupling (cw) was applied. (b) $\mathrm{F}_{1}$ projections of the isotropic center bands from the two-dimensional homonuclear ${ }^{31} \mathrm{P}$ J-resolved spectra of 1 , recorded at the indicated temperatures. Projections for P1 and P2 are shown in black and red, respectively.

with isotropic projections acquired at three temperatures. The spectrum was acquired using the pulse sequence given in ref 21 . At $298 \mathrm{~K}$, there is a clear splitting (of $\sim 86 \mathrm{~Hz}$ ) for both P1 and $\mathrm{P} 2$, suggesting a homonuclear J coupling between the two. This is in good agreement with the splitting observed for both resonances in the ${ }^{31} \mathrm{P}$ MAS spectrum at lower field. As there is only one $\mathrm{P}$ within each molecule this interaction must be a homonuclear intermolecular through-space coupling. This conclusion is supported by the short internuclear distance between the P sites (3.699 $\AA$ ) in the crystal structure (Figure $1 \mathrm{~b})$, close to the sum of their van der Waals radii.

The presence of a homonuclear intermolecular $\mathrm{J}$ coupling is confirmed by periodic DFT calculations, given in Table 2 . The calculated coupling $\left(J_{\mathrm{PP}}^{\text {calc }}\right)$ is $144 \mathrm{~Hz}$, rising to $151 \mathrm{~Hz}$ with the inclusion of scalar relativity (implemented using the ZORA ${ }^{19}$ 
approach). The total $\mathrm{J}$ coupling is dominated by the Fermicontact contribution, of $150 \mathrm{~Hz}$, at the scalar-relativistic level of theory. The calculated coupling is significantly larger than the value measured experimentally. However, given the throughspace nature of the interaction, the magnitude of this coupling will be crucially dependent upon the distance between the nuclear species. ${ }^{18,19}$ The difficulties of accurately reproducing dispersion interactions in periodic DFT calculations may lead to some variation in the exact distances between atoms and, although the D2 dispersion correction scheme of Grimme ${ }^{27}$ was employed in the DFT calculations, this could contribute to the differences between experiment and calculation. It is possible that differences between calculation and experiment could also result from the choice of functional. However, calculations to test the use of a hybrid functional (PBE0, containing $25 \%$ Hartree-Fock exchange) using a cluster approach show little change ( $3 \%$ between PBE and PBE0) in the calculated J couplings, which all remain larger than experiment (see the Supporting Information). Furthermore, Figure 2 shows that the experimental $\mathrm{J}$ coupling is temperaturedependent, with an increase of $2-3 \mathrm{~Hz}$ per $25 \mathrm{~K}$ temperature decrease. If this variation were linear over the whole temperature range, the predicted coupling at $0 \mathrm{~K}$ for $\mathbf{1}$ would be $\sim 110 \mathrm{~Hz}$, in slightly better agreement with calculation. Figure 3a shows a plot of the calculated $\mathrm{CDD}^{20}$ associated with
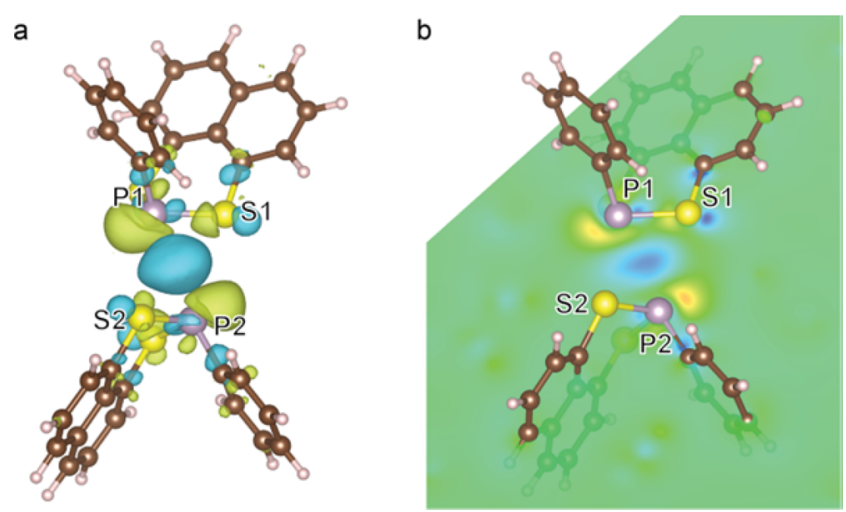

Figure 3. (a) Isosurface and (b) contour representations of the CDD of the ${ }^{31} \mathrm{P}-{ }^{31} \mathrm{P}$ through-space $\mathrm{J}$ couplings (computed from nonrelativistic J-coupling calculations) for $\mathbf{1}$.

the $\mathrm{P}-\mathrm{P}$ homonuclear $\mathrm{J}$ coupling in $\mathbf{1}$ (based on non-relativistic J-coupling calculations; see the Supporting Information). This supports the presence of a through-space interaction between $\mathrm{P} 1$ and $\mathrm{P} 2$ and shows that the coupling proceeds via the P lone pairs.

Naphtho[1,8-cd]-1,2-diselenole tert-butylphosphine (2) and Naphtho[1,8-cd]-1,2-diselenole isopropylphosphine (3). The ${ }^{31} \mathrm{P}$ MAS NMR spectra of 2 and 3 , acquired at 14.1 $\mathrm{T}$, are shown in Figure 4. In each case a single isotropic resonance (shown by $*$ ) is observed, in agreement with the presence of a single crystallographically distinct molecule in the asymmetric unit. Values of the isotropic and anisotropic shielding parameters extracted from the spectra are given in Table 1 . The change in the $\mathrm{R}$ group (from tert-butyl to isopropyl) results in a small change in the ${ }^{31} \mathrm{P}$ isotropic shift, and a larger change (of $\sim 34 \mathrm{ppm}$ ) in $\Omega$. As shown in the Supporting Information, the isotropic shift for both compounds is also temperature-dependent, with increases of $\sim 1$ and $\sim 0.5$

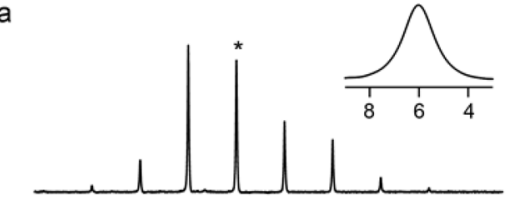

b


Figure 4. (a, c) ${ }^{31} \mathrm{P}$ (9.4 T, $7.5 \mathrm{kHz}$ MAS) NMR spectra of (a) 2 and (c) 3 , where $*$ denotes the isotropic resonances (shown expanded in the insets). (b, d) The asymmetric units of (b) 2 and (d) 3.

ppm per $25 \mathrm{~K}$ for 2 and 3, respectively, probably resulting from motion of the alkyl groups.

DFT-calculated NMR parameters for each compound are given in Table 2 . As was observed for $\mathbf{1}$, the calculated $\Omega$ is overestimated for both $\mathbf{2}$ and 3 (although the relative magnitude of the two values is well-reproduced), possibly as a result of the neglect of any motion in the calculation. The relative difference in isotropic shift is also in good agreement with experiment, although it should be noted that there is a significant discrepancy in the relative isotropic shieldings predicted for the S-containing and the two Se-containing compounds, perhaps suggesting a difference in accuracy between the heavy-atom pseudopotentials. While ${ }^{77} \mathrm{Se}$ DFT calculations have been shown to have good agreement with experiment in similar naphthalene-based systems, ${ }^{32,33}$ there has been little experimental verification of any predicted ${ }^{33} \mathrm{~S}$ NMR parameters, owing to the difficulties associated with experimental study of this low- $\gamma$, low-abundance quadrupolar nucleus.

Figure 5 shows two-dimensional ${ }^{31} \mathrm{P}$ homonuclear J-resolved spectra of 2 and 3, recorded at $14.1 \mathrm{~T}$ at $298 \mathrm{~K}$. A clear splitting
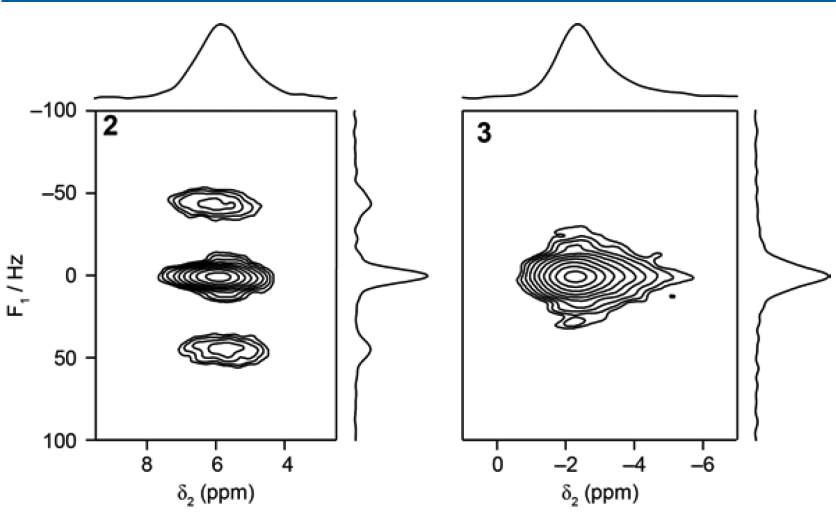

Figure 5. Expansions showing the isotropic center bands from the two-dimensional homonuclear ${ }^{31} \mathrm{P}$ J-resolved spectra of 2 and 3 (14.1 $\mathrm{T}, 5 \mathrm{kHz}$ MAS). ${ }^{1} \mathrm{H}$ decoupling (cw) was applied.

is observed for 2 , although an intense central signal at $0 \mathrm{~Hz}$ is also observed. As for $\mathbf{1}$, there is only one $\mathrm{P}$ within each molecule, and so this interaction must also be a homonuclear intermolecular through-space coupling. Note that no splitting is observed in the J-resolved spectrum of $\mathbf{3}$, despite the chemical 
similarity of the two molecules. However, this observation is also supported by the DFT calculations (Table 2), where a large $\mathrm{J}$ coupling is predicted for $2(159 \mathrm{~Hz})$, and a much smaller one for $3(11 \mathrm{~Hz})$. The Fermi-contact term is again dominant, at 160 and $11 \mathrm{~Hz}$ in 2 and 3, respectively. The predicted coupling for $\mathbf{2}$ is again larger than that observed experimentally $(\sim 88 \mathrm{~Hz})$ but, as Figure 6a shows, the coupling is temperature-

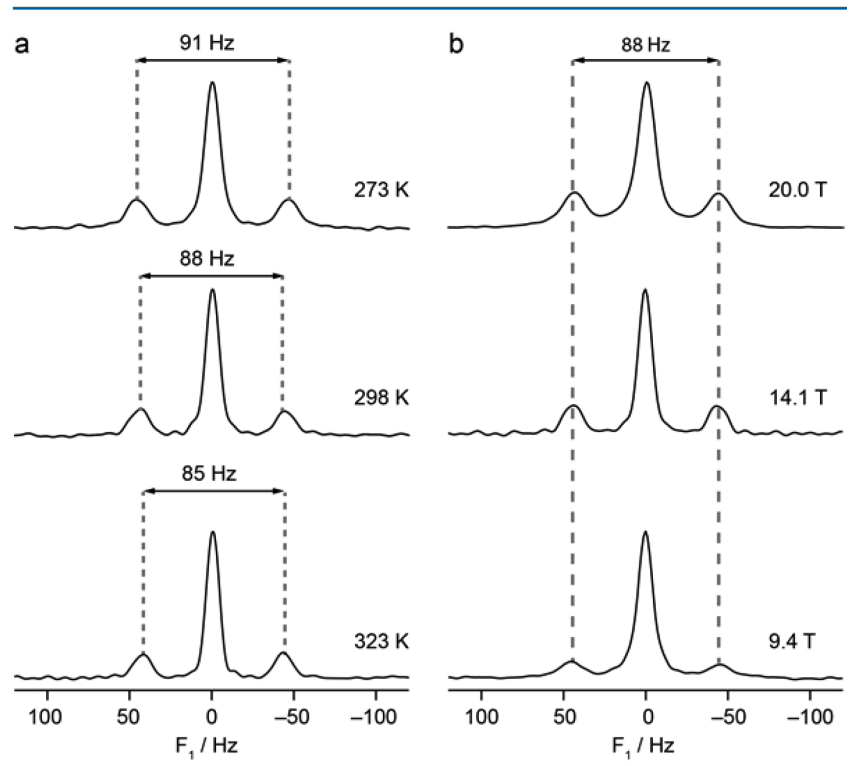

Figure 6. $F_{1}$ projections of the isotropic center bands from the twodimensional homonuclear ${ }^{31} \mathrm{P}$ J-resolved spectra of 2 , recorded at (a) 14.1 $\mathrm{T}$ at the temperatures indicated and (b) at room temperature at the $B_{0}$ field indicated.

dependent (increasing by $3 \mathrm{~Hz}$ per $25 \mathrm{~K}$ decrease in temperature). If this change could be assumed to be linear over the whole temperature range, the predicted $0 \mathrm{~K}$ coupling for 2 would be $\sim 125 \mathrm{~Hz}$, in better agreement with the calculation. Given the through-space nature of the interactions observed, the difference in the coupling magnitude between 2 and 3 can be explained by the different intermolecular $\mathrm{P}-\mathrm{P}$ distances, which, from the single-crystal X-ray diffraction structures (Figure 4b), vary from $3.58 \AA$, for 2 , to $4.90 \AA$ for 3 . $^{18}$ The former is within the sum of the van der Waals radii, while the latter is outside this range. The CDD plots, shown in Figure 7, demonstrate that the $\mathrm{J}$ coupling in $\mathbf{2}$ is, again, mediated by the $\mathrm{P}$ lone-pair electrons, while no clear interaction between the two $\mathrm{P}$ atoms is present for 3 .

The experimental observation of a ${ }^{31} \mathrm{P}-{ }^{31} \mathrm{P}$ coupling for $\mathbf{2}$ is particularly unexpected, as this interaction must occur between crystallographically equivalent $P$ species, that is, species with the same isotropic chemical shift. It is well-known that it is not possible to observe a $\mathrm{J}$ coupling between equivalent spins in a solution-state NMR spectrum. Similarly, one would not expect to see any splitting in a J-resolved spectrum, which should consist of only a central (i.e., unmodulated) signal. However, in the solid state, crystallographically equivalent spins need not be magnetically equivalent. ${ }^{34}$ If anisotropic interactions are present (e.g., a CSA), the magnitude and asymmetry of these will be the same for crystallographically equivalent spins, but the relative orientation of the tensors may be different, owing to the different orientation of the molecules with respect to $B_{0}$. In this case, the anisotropic shifts would be different for each molecule, lifting any magnetic equivalence at any one instant during the a
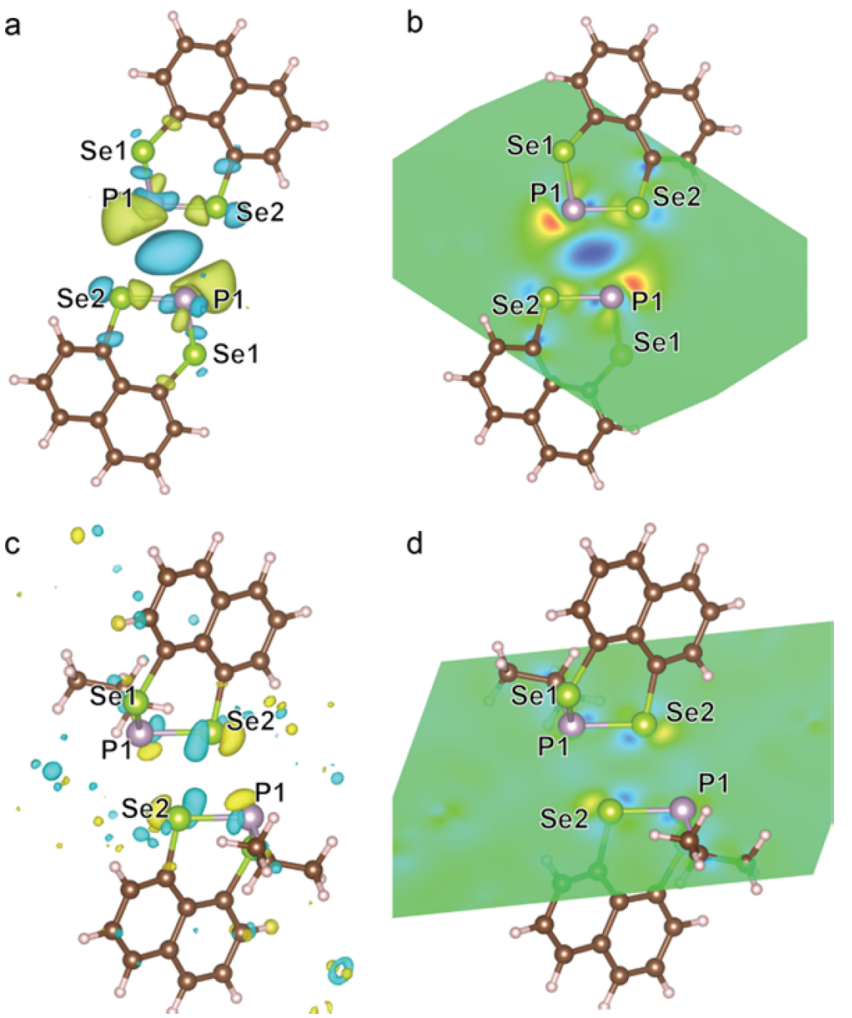

Figure 7. (a, c) Isosurfaces and (b, d) contour representations of the $\mathrm{CDD}$ of the ${ }^{31} \mathrm{P}-{ }^{31} \mathrm{P}$ through-space $\mathrm{J}$ couplings (computed from nonrelativistic J-coupling calculations) for (a, b) 2 and (c, d) 3.

rotor period, and a $\mathrm{J}$ coupling could then be measured. ${ }^{31}$ Reference 31 discussed in detail the effects this would have on the spin-echo modulation and, therefore, on a J-resolved experiment, and demonstrated that this depends upon magnitude of the anisotropy, the MAS rate, and the magnitude of the J coupling. However, although there is a significant anisotropic contribution to the ${ }^{31} \mathrm{P}$ shielding for $2(\Omega \approx 231$ $\mathrm{ppm}$ ), the two ${ }^{31} \mathrm{P}$ species involved in the coupling (and, therefore, their CSA tensors) are related via an inversion center, ${ }^{18}$ resulting in both crystallographic and magnetic equivalence. It should also be noted that, as shown in Figure $6 \mathrm{~b}$, there is no significant change in the J-resolved spectrum of 2 with varying magnetic field strength, which might be expected if an anisotropic interaction were responsible for lifting magnetic equivalence.

Although a homonuclear ${ }^{31} \mathrm{P}-{ }^{31} \mathrm{P}$ coupling is observed for $\mathbf{2}$, the explanation for the appearance of the J-resolved spectrum may lie in the heteronuclear couplings between ${ }^{31} \mathrm{P}$ and ${ }^{77} \mathrm{Se}$. As shown in Figure 8, the two $\mathrm{P}$ species are each bonded to two Se nuclei. The natural abundance of the only NMR-active isotope $\left({ }^{77} \mathrm{Se}, I=1 / 2\right)$ is $\sim 7.6 \%$. If all four Se in the two molecules in Figure 8 are NMR inactive, the two ${ }^{31} \mathrm{P}$ species are magnetically equivalent, and no splitting will be observed in a J-resolved spectrum. However, if only one of the four were ${ }^{77} \mathrm{Se}$, there would be a through-bond ${ }^{77} \mathrm{Se}-{ }^{31} \mathrm{P}$ coupling experienced by one ${ }^{31} \mathrm{P}$ (e.g., as shown in Figure 8) that is not present for the second, resulting in magnetic inequivalence of the two $\mathrm{P}$ species. When this coupling is present, the homonuclear ${ }^{31} \mathrm{P}-{ }^{31} \mathrm{P}$ interaction then becomes observable. Simple statistics predicts that the probability of magnetic equivalence (i.e., no ${ }^{77}$ Se bonded to either ${ }^{31} \mathrm{P}$ ) is $\sim 73 \%$, while $\sim 27 \%$ of the pairs will have one or more ${ }^{77} \mathrm{Se}$ present (and, hence, result in 
a

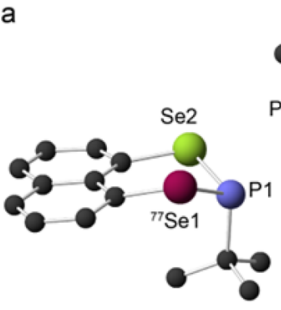

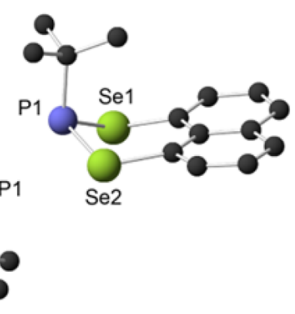

b

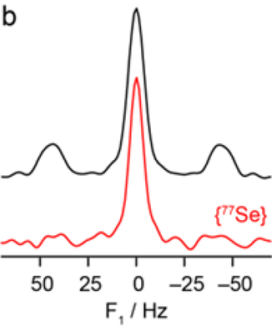

Figure 8. (a) Expansion of the structure of 2, with a possible ${ }^{77} \mathrm{Se} /{ }^{31} \mathrm{P}$ heteronuclear J coupling highlighted. (b) Projections (indirect dimension) of two-dimensional ${ }^{31} \mathrm{P}(14.1 \mathrm{~T}, 5 \mathrm{kHz})$ homonuclear Jresolved MAS NMR spectra of 2 , acquired with (red) and without (black) ${ }^{77}$ Se decoupling.

magnetic inequivalence). This should result in a combination of signals in the J-resolved spectrum from $73 \%$ of molecules, where no coupling is observed (and a central signal is expected to be present), and $27 \%$, where a splitting is seen, in good agreement with the experimental spectra in Figures $5 a$ and 6. Note, however, that for a single ${ }^{77} \mathrm{Se}$, there are two possible Se sites to consider. If $\mathrm{Se} 2$ is ${ }^{77} \mathrm{Se}$, in addition to the ${ }^{77} \mathrm{Se} 2-{ }^{31} \mathrm{P} 1$ through-bond coupling (estimated in previous work to be $\sim 270$ $\mathrm{Hz}$ ), there is a significant through-space intermolecular heteronuclear ${ }^{77} \mathrm{Se} 2-{ }^{31} \mathrm{P} 1$ coupling (estimated previously to be $\sim 340 \mathrm{~Hz}) .{ }^{18}$ However, if $\mathrm{Se} 1$ is ${ }^{77} \mathrm{Se}$, the through-space coupling to ${ }^{31} \mathrm{P}$ in the neighboring molecule is much smaller (not observed experimentally). Despite the similarity in the magnitude of the through-bond and through-space ${ }^{77} \mathrm{Se} 2-{ }^{31} \mathrm{P} 1$ couplings, ${ }^{18}$ the $\mathrm{J}$ coupling tensors have different orientations, and the two ${ }^{31} \mathrm{P}$ remain inequivalent even if these additional couplings are considered. Note that the ${ }^{77} \mathrm{Se}-{ }^{31} \mathrm{P}$ couplings are not resolved in the ${ }^{31} \mathrm{P}$ MAS spectrum in Figure 4, owing to the large inherent line width $(\sim 350 \mathrm{~Hz})$ and the low natural abundance of ${ }^{77} \mathrm{Se}$.

To ascertain whether the magnetic inequivalence that must be present for the J coupling to be observable does arise from heteronuclear couplings, ${ }^{77} \mathrm{Se}$ decoupling was implemented in the indirect dimension of the J-resolved experiment for 2 . The large ${ }^{77}$ Se CSA observed for 2 in previous work ${ }^{18}$ suggests that high-power decoupling would be required. However, owing to the hardware available, it was not possible to use high-power $\mathrm{cw}$ decoupling for the long evolution periods required, and a pulsed decoupling approach ${ }^{22}$ was used (see the Experimental Section). Figure $8 \mathrm{~b}$ shows the center band projections of twodimensional ${ }^{31} \mathrm{P}$ homonuclear J-resolved spectra of 2 , acquired with and without ${ }^{77} \mathrm{Se}$ decoupling. ${ }^{1} \mathrm{H}$ cw decoupling was also applied in both cases. It can be seen that the homonuclear ${ }^{31} \mathrm{P} /{ }^{31} \mathrm{P} \mathrm{J}$ coupling is not observed when the $\mathrm{J}$ coupling to ${ }^{77} \mathrm{Se}$ is removed by decoupling, with only the unmodulated central signal present, confirming it is this heteronuclear interaction that lifts the magnetic equivalence of the two P sites.

One final consideration is the temperature dependence of the NMR parameters observed for $\mathbf{2}$. There is clearly significant molecular motion in the compound, potentially of both the tertbutyl and $\mathrm{CH}_{3}$ groups. In principle, unless this motion is concerted (or very rapid), this could also break the inversion symmetry and lift the equivalence of the two ${ }^{31} \mathrm{P}$ species. This could lead to differences in isotropic or anisotropic shielding parameters for the two coupled $\mathrm{P}$ species and might result in the observation of a splitting in a J-resolved spectrum, even for molecules where no couplings to ${ }^{77} \mathrm{Se}$ are present. However, both the loss of the splitting upon ${ }^{77}$ Se decoupling in Figure 8

and the lack of any field dependence of the J-resolved spectrum in Figure $6 \mathrm{~b}$ suggest that motion is not the primary cause of the inequivalence observed at the temperatures considered.

\section{CONCLUSIONS}

We have demonstrated the observation of unusual intermolecular homonuclear through-space $\mathrm{J}$ couplings between $\mathrm{P}$ species in different molecules in the solid state. Although through-space couplings have been seen in the literature previously, most of these have been in solution-state NMR spectra, between atoms that are forced into close proximity by a rigid molecular backbone. The rapid molecular tumbling in solution restricts the observation of such couplings to those between atoms within a molecule, while the solid-state crystal packing enables couplings to be observed between different molecules. The presence of only one $\mathrm{P}$ species per organochalcogen molecule validates unambiguously the through-space, rather than conventional through-bond, nature of these interactions. For 1, the coupling is observed between crystallographically distinct $\mathrm{P}$ species, but the observation is more unexpected for 2 , where the two $\mathrm{P}$ species are crystallographically equivalent. However, we have demonstrated that the presence of heteronuclear J couplings to ${ }^{77} \mathrm{Se}(7.6 \%$ abundance) lifts magnetic equivalence in some molecules, enabling a direct observation of the coupling. The increased observation of through-space couplings in molecular solids perhaps suggests that these interactions need to be considered in the context of the factors that affect crystal packing and also offer further insight both into understanding the origin of $\mathrm{J}$ couplings and into the future design of solid-state structures.

\section{ASSOCIATED CONTENT}

\section{S Supporting Information}

The Supporting Information is available free of charge on the ACS Publications website at DOI: 10.1021/acs.inorgchem.6b01121.

Full synthesis and characterization of 1 , variable-temperature ${ }^{31} \mathrm{P}$ NMR spectra of $1-3$, and extended details of the calculations (PDF)

\section{AUTHOR INFORMATION}

\section{Corresponding Authors}

*E-mail: sema@st-andrews.ac.uk. (S.E.A.)

*E-mail: jdw3@st-andrews.ac.uk. (J.D.W.)

\section{Author Contributions}

The manuscript was written through contributions of all authors. All authors have given approval to the final version of the manuscript.

\section{Notes}

The authors declare no competing financial interest.

\section{ACKNOWLEDGMENTS}

We would like to thank the ERC (EU FP7 Consolidator Grant No. 614290 "EXONMR") and EPSRC for computational support through the Collaborative Computational Project on NMR Crystallography (CCP-NC), via EP/M022501/1 and EP/J501542/1. S.E.A. would like to thank the Royal Society and the Wolfson Foundation for a merit award. We gratefully acknowledge support from the Leverhulme Trust (IN-2012094). We would also like to acknowledge Prof. D. Bryce for helpful discussions. The UK $850 \mathrm{MHz}$ solid-state NMR Facility 
used in this research was funded by EPSRC and BBSRC (Contract No. PR140003), as well as the Univ. of Warwick including via part funding through Birmingham Science City Advanced Materials Projects 1 and 2 supported by Advantage West Midlands and the European Regional Development Fund. Collaborative assistance from the $850 \mathrm{MHz}$ Facility Manager (Dinu Iuga, Univ. of Warwick) is acknowledged. J-coupling calculations for $\mathbf{1}$ were performed on the ARCHER UK National Supercomputing Service and were supported by CCPNC. The research data (and/or materials) supporting this publication can be accessed at DOI: http://dx.doi.org/10. 17630/6871d21d-e523-4946-9445-2a95e923d6e9.

\section{REFERENCES}

(1) Karplus, M. J. Chem. Phys. 1959, 30, 11-15.

(2) Hierso, J. C. Chem. Rev. 2014, 114, 4838-4867.

(3) Hierso, J. C.; Armspach, D.; Matt, D. C. R. Chim. 2009, 12, $1002-1013$.

(4) Saika, A.; Gutowsky, H. S. J. Am. Chem. Soc. 1956, 78, 48184819.

(5) Bryce, D. L.; Wasylishen, R. E. J. Mol. Struct. 2002, 602, 463-472. (6) Aschenbach, L. K.; Knight, F. R.; Randall, R. A M.; Cordes, D. B.; Baggott, A.; Bühl, M.; Slawin, A. M. Z.; Woollins, J. D. Dalton Trans. 2012, 41, 3141-3153.

(7) Bühl, M.; Knight, F. R.; Kř́stková, A.; Malkin Ondík, I.; Malkina, O. L.; Randall, R. A. M.; Slawin, A. M. Z.; Woollins, J. D. Angew. Chem., Int. Ed. 2013, 52, 2495-2498.

(8) Contreras, R. H.; Ducati, L. C.; Tormena, C. F. Int. J. Quantum Chem. 2012, 112, 3158-3163.

(9) Dracinsky, M.; Jansa, P.; Bour, P. Chem. - Eur. J. 2012, 18, 981986.

(10) Contreras, R. H.; Gotelli, G.; Ducati, L. C.; Barbosa, T. A.; et al. J. Phys. Chem. A 2010, 114, 1044-1051.

(11) Wiegand, T.; Eckert, H.; Ren, J.; Brunklaus, G.; Fröhlich, R.; Daniliuc, C. G.; Lübbe, G.; Bussmann, K.; Kehr, G.; Erker, G.; Grimme, S. J. Phys. Chem. A 2014, 118, 2316-2331.

(12) Wiegand, T.; Eckert, H.; Ekkert, O.; Fröhlich, R.; Kehr, G.; Erker, G.; Grimme, S. J. Am. Chem. Soc. 2012, 134, 4236-4249.

(13) Wu, G.; Wasylishen, R. E.; Pan, H.; Liu, C. W.; Fackler, J. P.; Shang, M. Magn. Reson. Chem. 1995, 33, 734-738.

(14) Stanford, M. W.; Knight, F. R.; Athukorala Arachchige, K. S.; Sanz Camacho, P.; Ashbrook, S. E.; Bühl, M.; Slawin, A. M. Z.; Woollins, J. D. Dalton Trans. 2014, 43, 6548-6560.

(15) Knight, F. R.; Diamond, L. M.; Athukorala Arachchige, K. S.; Sanz Camacho, P.; Randall, R. A. M.; Ashbrook, S. E.; Bühl, M.; Slawin, A. M. Z.; Woollins, J. D. Chem. - Eur. J. 2015, 21, 3613-3627.

(16) Griffin, J. M.; Yates, J. R.; Berry, A. J.; Wimperis, S.; Ashbrook, S. E. J. Am. Chem. Soc. 2010, 132, 15651-15660.

(17) Brown, S. P.; Perez-Torralba, M.; Sanz, D.; Claramunt, R. M.; Emsley, L. J. Am. Chem. Soc. 2002, 124, 1152-1153.

(18) Sanz Camacho, P.; Athukorala Arachchige, K. S.; Slawin, A. M. Z.; Green, T. F. G.; Yates, J. R.; Dawson, D. M.; Woollins, J. D.; Ashbrook, S. E. J. Am. Chem. Soc. 2015, 137, 6172-6175.

(19) Arras, J.; Eichele, K.; Maryasin, B.; Schubert, H.; Ochsenfeld, C.; Wesemann, L. Inorg. Chem. 2016, 55, 4669-4675.

(20) Malkina, O. L.; Malkin, V. G. Angew. Chem., Int. Ed. 2003, 42, 4335-4338.

(21) Brown, S. P.; Perez-Torralba, M.; Sanz, D.; Claramunt, R. M.; Emsley, L. Chem. Commun. 2002, 1852-1853.

(22) Griffin, J. M.; Tripon, C.; Samoson, A.; Filip, C.; Brown, S. P. Magn. Reson. Chem. 2007, 45, 198-208.

(23) Skibsted, J.; Jakobsen, H. J. J. Phys. Chem. A 1999, 103, 79587971.

(24) Clark, S. J.; Segall, M. D.; Pickard, C. J.; Hasnip, P. J.; Probert, M. J.; Refson, K.; Payne, M. C. Z. Kristallogr. - Cryst. Mater. 2005, 220, 567-570.

(25) Pickard, C. J.; Mauri, F. Phys. Rev. B: Condens. Matter Mater. Phys. 2001, 63, 245101.
(26) Perdew, J. P.; Burke, K.; Ernzerhof, M. Phys. Rev. Lett. 1996, 77, 3865-3868.

(27) Grimme, S. J. Comput. Chem. 2006, 27, 1787-1799.

(28) McNellis, E. R; Meyer, J.; Reuter, K. Phys. Rev. B: Condens. Matter Mater. Phys. 2009, 80, 205414.

(29) Autschbach, J.; Ziegler, T. J. Chem. Phys. 2000, 113, 9410-9418.

(30) Fuller, A. L.; Knight, F. R.; Slawin, A. M. Z.; Woollins, J. D. Eur. J. Inorg. Chem. 2010, 2010, 4034-4043.

(31) Duma, L.; Lai, W. C.; Carravetta, M.; Emsley, L.; Brown, S. P.; Levitt, M. H. ChemPhysChem 2004, 5, 815-833.

(32) Bonhomme, C.; Gervais, C.; Babonneau, F.; Coelho, C.; Pourpoint, F.; Azaïs, T.; Ashbrook, S. E.; Griffin, J. M.; Yates, J. R.; Mauri, F.; Pickard, C. J. Chem. Rev. 2012, 112, 5733-5779.

(33) Griffin, J. M.; Knight, F. R.; Hua, G.; Ferrara, J. S.; Hogan, S. W. L.; Woollins, J. D.; Ashbrook, S. E. J. Phys. Chem. C 2011, 115, 1085910872.

(34) Perras, F.; Bryce, D. L. eMagRes. 2015, 561-574. 